\title{
MÁS ALLÁ DE LOS “DATOS”: UNA BREVE REFLEXIÓN DESDE LA EPIDEMIOLOGÍA SOCIAL A LOS SISTEMAS TRADICIONALES DE INFORMACIÓN Y VIGILANCIA DE LA VIOLENCIA
}

\section{BEYOND THE "DATA": A BRIEF REFLECTION FROM SOCIAL EPIDEMIOLOGY TO TRADITIONAL SYSTEMS OF INFORMATION AND SURVEILLANCE OF VIOLENCE}

\author{
Andrés Castillo Vargas*
}

RESUMEN

\begin{abstract}
Este artículo tiene como propósito principal presentar algunas reflexiones teóricas sobre las características, funciones e importancia de la epidemiología social en los sistemas tradicionales de información y vigilancia de la violencia. Con tal objetivo, se aborda la violencia como un problema de salud pública, que trasciende los factores numéricos y converge con variables socioculturales e históricas, incorporando en su abordaje aspectos teóricos y metodológicos de la epidemiología social. Los resultados de esta reflexión permiten contemplar tanto la relevancia como la necesidad de que los organismos encargados de diseñar y poner en práctica los sistemas de información, conozcan a profundidad las propuestas de la epidemiología social. A manera de conclusión, este trabajo propone que las problemáticas sociales de salud pública sean contempladas desde la perspectiva integral y colectiva de los procesos humanos que plantea la epidemiología social.
\end{abstract}

PALABRAS CLAVE: EPIDEMIOLOGÍA * SALUD * SISTEMAS DE INFORMACIÓN * VIOLENCIA * VIGILANCIA * SALUD PÚBLICA

\section{ABSTRACT}

The main purpose of this article is to present some theoretical reflections on the characteristics, functions and importance of social epidemiology in traditional systems of information and surveillance of violence. With this objective, violence is addressed as a public health problem, which transcends numerical factors and converges with sociocultural and historical variables, incorporating theoretical and methodological aspects of social epidemiology. The results of this reflection allow us to contemplate both the relevance and the need for the agencies in charge of designing and putting into

* Escuela de Psicología, Universidad de Costa Rica, Costa Rica. andres.castillo@ucr.ac.cr 
practice the information systems, to know in depth the proposals of social epidemiology. As a conclusion, this work proposes that the social problems of public health be considered from the integral and collective perspective of the human processes posed by social epidemiology.

KEYWORDS: EPIDEMIOLOGY * HEALTH * INFORMATION SYSTEMS * VIOLENCE * SURVEILLANCE * PUBLIC HEALTH

\section{INTRODUCCIÓN}

La categorización de la violencia como un claro problema de salud pública, en tanto "afecta de manera grave la salud y el desarrollo social y económico de amplios sectores de la población" (Concha-Eastman y Villaveces, 2001, p. 91), fue uno de los principales factores que motivaron la implementación progresiva de sistemas de información orientados a registrar, tanto sus manifestaciones como su incidencia; así como, otras dimensiones, cuya comprensión orientara la toma de decisiones respecto a su abordaje.

Este reconocimiento gestó cambios importantes no solo en términos de una mayor conciencia e interés respecto a la problemática, sino que también coincidió con un proceso de ampliación del campo de acción de la epidemiología - que algunos sectores venían desarrollando-, con miras a validar la inclusión de la dimensión social en sus análisis. Dentro de este contexto, la implementación de sistemas de vigilancia epidemiológica que transciendan los datos duros, constituyen un avance significativo en la expansión de los horizontes desde los cuales se significa la salud pública y en la comprensión de las necesidades de determinados grupos humanos.

Ante la apertura de estos nuevos espacios de interrelación, legitimación y preocupación social, la presente reflexión plantea una breve revisión tanto de las principales características y funciones de los sistemas de vigilancia epidemiológica, así como de las nuevas posibilidades $y$ debates que abre la epidemiología social —como subdisciplina de la epidemiología - para el mejoramiento de las oportunidades $y$ la calidad de dichos sistemas.
En aras de garantizar la comprensión de esta temática, en los siguientes apartados se expone inicialmente el vínculo entre la salud pública y el fenómeno de la violencia, así como, las características y las limitaciones de los sistemas de vigilancia de la salud. Seguidamente, se retoman las contribuciones realizadas por la epidemiología social al mejoramiento de los sistemas de información y vigilancia, al transitar de la realidad numérica a la realidad social en el análisis de datos.

Se espera que las siguientes líneas contribuyan al mejoramiento de la respuesta estatal e institucional en torno a la recopilación, sistematización y análisis de datos vinculados al ejercicio del derecho a la salud de grupos humanos específicos.

\section{VIOLENCIA Y SALUD PÚBLICA}

La violencia ha sido construida socialmente $y$ determinada por las condiciones de vida de las personas. En la actualidad, está presente en todas las sociedades $y$ en diferentes manifestaciones, las cuales varían de significado según el contexto en el cual se producen (Organización Panamericana de la Salud-oPS, 2004).

La Organización Mundial de la Salud (oms) define a la violencia como el uso intencional de la fuerza o el poder físico contra uno mismo, otra persona o un grupo o comunidad, que cause o tenga probabilidades de causar lesiones, muerte, daños psicológicos, trastornos del desarrollo o privaciones. Es dividida en tres categorías generales, según las características de quienes cometen el acto de violencia: violencia auto infligida (suicidio y autolesiones), violencia interpersonal (violencia familiar, de 
pareja y violencia comunitaria) y violencia colectiva (social, política y económica) (oMs, 2002).

La salud pública ha considerado la violencia como un problema porque ocasiona gran cantidad de muertes, perjudicando en su mayoría a la niñez, las mujeres y las personas jóvenes (grupos social e históricamente vulnerabilizados); esta violencia también produce efectos negativos en las familias de las víctimas $y$ en su entorno. Asimismo, las víctimas de violencia demandan elevados gastos en la atención médica, produciendo impactos negativos en el desarrollo social y económico de las comunidades y países (Concha, 2002).

Parafraseando a Larizgoitia (2006), la violencia es un problema que debe ser abordado a partir de una visión amplia de lo que es la salud pública, pues desde una perspectiva social son múltiples sus determinantes, los cuales vislumbran la necesidad y el compromiso de ampliar la comprensión sanitaria tradicional de la salud, complementándola con una visión inclusiva de los aspectos sociales, culturales $y$ contextuales.

A través de una visión comunitaria y contextual de la salud pública se puede comprender que la violencia afecta el bienestar de las personas, tanto a nivel individual como colectivo; es así como la epidemiología busca aportar metodologías novedosas que permitan caracterizar al problema de la violencia, identificar sus determinantes $y$ visualizar el impacto que tiene en los colectivos humanos, con miras a facilitar su comprensión y fortalecer las posibilidades de acción para hacerle frente (Larizgoitia, 2006).

\section{VIGILANCIA DE LA SALUD: CARACTERÍSTICAS Y LIMITACIONES}

El concepto de vigilancia epidemiológica fue introducido en 1955 por el Centro de Enfermedades Transmisibles del Servicio de Salud Pública de los Estados Unidos; posteriormente, a este término se le incorporaron componentes del área de la salud pública, permitiendo que actualmente se le conozca también como vigilancia de la salud (Lemus, 1996).

La vigilancia de la salud fue conceptualizada como una rama de la epidemiología, aunque actualmente es considerada una disciplina independiente en el campo de la salud pública. Esta es entendida como "la observación sistemática y continuada de la frecuencia, la distribución y los determinantes de los eventos de salud y sus tendencias en la población" (OPS, 2011, p. 6), de modo que analiza, interpreta y difunde sistemáticamente los datos recolectados por diversos sistemas de información, para realizar acciones, investigaciones $y / 0$ aplicaciones de medidas de control de enfermedades para mejorar la calidad de vida y de salud de las personas.

La OPs (2011) considera que los usos de la vigilancia de la salud son de tres tipos, a saber:

$\diamond \quad$ Seguimiento de los eventos de salud: estima la magnitud de los eventos, detecta la distribución de las enfermedades, las tendencias del proceso salud-enfermedad y los cambios en las prácticas de salud.

$\diamond \quad$ Acciones de salud pública: permite que se investigue y controlen las enfermedades a partir de los datos recolectados, planea los programas de salud y evalúa las medidas de control y prevención.

$\diamond \quad$ Otros usos: permite probar hipótesis generadas por el análisis de los datos y tener archivos históricos de la actividad de las enfermedades.

Tomando en consideración los usos anteriores y que todo sistema de vigilancia debe contar con un marco legal propio, la OPS (2011) considera a la vigilancia en salud como un proceso continuo y sistemático, que investiga tendencias y que es de comparación, entre lo que se observa y lo que se espera. En este sentido, los sistemas de vigilancia epidemiológica permiten solucionar los vacíos existentes de información, respondiendo a interrogantes como ¿quiénes son las personas o poblaciones afectadas?, ¿en qué circunstancias y condiciones?, ¿cuándo ocurren los eventos? y ¿cuáles factores se asocian con su ocurrencia?; las cuales resultan de gran utilidad para el desarrollo de intervenciones, programas o proyectos para el 
control, atención y prevención de enfermedades o eventos sociales como la violencia (OPS, 2001).

Los Sistemas de Vigilancia Epidemiológica (SVE) responden a la necesidad — gestada inicialmente desde el área de la salud públicade solucionar vacíos de información respecto a temáticas de interés específicas, bajo criterios claramente definidos que buscan asegurar la calidad de los datos producidos. Tales sistemas sobresalen, en consecuencia, como una herramienta de trabajo indispensable para la epidemiología (Oakes y Kaufman, 2006).

Una de las características principales de los métodos de análisis y el valor predictivo de los sistemas de vigilancia epidemiológica es que no se aplican a individuos particulares, sino a poblaciones, respecto a las cuales interesa conocer la dinámica, magnitud, tendencias y particularidades de los fenómenos que les afectan, todo ello con miras a la toma de decisiones políticas, técnicas o de otra índole que atiendan de manera efectiva dichas problemáticas.

Al respecto, Concha-Eastman y Villaveces (2001) acotan que el análisis e interpretación de los datos debe proporcionar bases para la toma de decisiones $y$ al mismo tiempo, ser utilizada para su difusión e impacto en la salud de los grupos humanos. Así, tal y como lo dejan entrever dichos autores, los orígenes y el desarrollo de la epidemiología como disciplina, se encuentran estrechamente ligados y determinados por la ciencia médica, la cual legitimó su utilidad y relevancia en el estudio de los principales factores de morbilidad y mortalidad que aquejaban a determinados colectivos.

Justamente esta vinculación con la práctica médica, que marca el origen histórico de la epidemiología, constituye una variable fundamental para comprender por qué aún en la actualidad, en muchos sistemas de información y vigilancia epidemiológica, se contemplan prioritariamente datos referidos a aspectos biológicos (epidemiología clínica) o datos numérico-estadísticos (epidemiología clásica), en detrimento de otros factores sociales que confluyen en calidad de determinantes de aquellos patrones y relaciones que la epidemiología estudia (González, 2000; Drucker, 2006).
Tal limitación es identificada por González (2000), al analizar algunas de las principales características de los sistemas de información en salud mexicanos, en los cuales "el acento en los logros $y$ en las metas a alcanzar en el campo de la población y de la salud se mantiene reducido a una valoración estadística" (p. 213), esto es, sin un análisis comprensivo que integre como elemento indispensable las variables socioculturales que inciden en la dinámica de todos los fenómenos y/o procesos vinculados con la salud humana y que, por consiguiente, requieren ser abordados por cualquier programa o intervención que les examine.

Lamentablemente, esta particularidad no es exclusiva del contexto mexicano y es bastante común en los sistemas de información epidemiológica de América Latina.

Ahora bien, aparte del énfasis en los "datos duros" o cuantitativos, otras limitaciones han sido identificadas en los sistemas de información existentes que recopilan y sistematizan registros en torno a la salud, $y$ en específico, a las diversas manifestaciones de la violencia; algunas de ellas son: la ligereza en la definición de variables, la no comparación de los datos con otras fuentes existentes, la no actualización del sistema en virtud de evaluaciones periódicas que determinen aspectos por mejorar o modificar, la ausencia de análisis basados en género o la omisión del enfoque generacional, contextual, de derechos humanos e interseccional en la dilucidación de los datos. Es así como la gran mayoría de las instituciones maneja información de manera aislada e independiente, sin que sea corroborada con otras entidades, lo cual origina que la información sea disímil y contradictoria.

En atención de estas áreas por fortalecer, Concha-Eastman y Villaveces (2001) insisten en la importancia de partir de una clara base conceptual respecto a los objetivos que persiguen este tipo de sistemas de monitoreo. En otras palabras, como fines primordiales, tales herramientas deben proponerse "producir información confiable y oportuna del problema en estudio, en sus variables sociodemográficas, características y circunstancias, analizada y entregada a quienes competa" (p. 14); así como 
"permitir el monitoreo de la magnitud y distribución de la morbilidad, mortalidad, efectos psicológicos, percepción y realidad del hecho, identificación de nuevos riesgos y formulación de posibles asociaciones causales" (ConchaEastman y Villaveces, 2001, p. 102).

Si bien, las anteriores funciones enfatizan en los focos de calidad, análisis y multidimensionalidad de los datos, los productos finales elaborados deben igualmente contribuir a la identificación de patrones $y$ tipologías, la generación de hipótesis sobre causalidad, la evaluación de impacto de un programa o intervención, así como, la conformación de grupos de trabajo interinstitucionales. De este modo, se revela la importancia que enviste para un sistema de información, el posicionamiento crítico respecto a los datos identificados, es decir, el procesamiento analítico de dicha información, en tanto será este el que podrá proponer la formulación de acciones concretas que conlleven una incidencia real en las problemáticas seleccionadas; es decir, no se trata solo de presentar cifras, sino de darles sentido y significado.

Dentro de este contexto de interpretación, adquiere relevancia señalar que la precisión en la elaboración de un sistema, así como, la rigurosidad y validación a la cual se someta cada uno de los pasos a seguir en su configuración, sobresalen como requisitos indispensables para asegurar la confiabilidad final de sus datos. No solo se sugiere que en el montaje de un sistema de vigilancia epidemiológica se debe partir de una clara definición de objetivos y variables, sino que también debe contemplar la conformación de un equipo de trabajo o comité técnico que brinde soporte y seguimiento al sistema (Concha-Eastman y Villaveces, 2001).

De igual modo, se plantea la importancia de identificar las fuentes de datos existentes para someterlas a un análisis minucioso de sus Fortalezas, Oportunidades, Debilidades y Amenazas (FODA), además de realizar una prueba piloto con la propuesta inicial del sistema antes de su entrada en vigor, comparar sus resultados con los provenientes de otras fuentes, definir la estrategia de difusión y/o divulgación apropiada y considerar la definición de acciones de prevención vinculadas, así como posibles alternativas de financiamiento.

En síntesis, garantizar la claridad y la precisión, pero también la comparación y la discusión en las diferentes fases de elaboración del sistema, favorece la calidad de sus datos y la consecuente pertinencia de sus recomendaciones. No obstante, ello debe también acompañarse de un diseño de evaluación definido que observe tanto la valoración de su utilidad, como de sus atributos, aunados a la descripción de los recursos humanos y económicos que se emplearían para operar el sistema (ConchaEastman y Villaveces, 2001).

Justamente, en lo que refiere a la valoración de los atributos de un sistema de vigilancia epidemiológica, deben definirse indicadores de evaluación para cada uno de ellos, tal como se ejemplifica en el cuadro 1. 
CUADRO 1

INDICADORES DE EVALUACIÓN DE UN SISTEMA DE VIGILANCIA EPIDEMIOLÓGICA (SVE)

\begin{tabular}{c|l}
\hline $\begin{array}{c}\text { ATRIBUTOS } \\
\text { DE UN SVE }\end{array}$ & \multicolumn{1}{c}{ CRITERIOS DE EVALUACIÓN } \\
\hline Simplicidad & $\begin{array}{l}\text { - Claridad en definiciones } \\
\text { - Selección de fuentes con aportes confiables } \\
\text { - Menor número de fuentes } \\
\text { - Costos de operación acordes con localidad o país }\end{array}$ \\
\hline Flexibilidad & $\begin{array}{l}\text { - Facilidad de adaptación a modificaciones operativas o estructurales } \\
\text { - Facilidad de respuesta a situaciones o riesgos nuevos }\end{array}$ \\
\hline Aceptabilidad & $\begin{array}{l}\text { - Compromiso de los grupos de trabajo y las instituciones } \\
\text { - Interacción de grupos en el funcionamiento del sve }\end{array}$ \\
\hline Sensibilidad & - Capacidad para detectar casos en comparación con otro método de mayor credibilidad \\
\hline Valor predictivo positivo & $\begin{array}{l}\text { - Confiabilidad de los datos } \\
\text { - Confirmación o descalificación de los casos definidos por el sve }\end{array}$ \\
\hline Representatividad & - Características del sve y de la población \\
\hline Oportunidad & - Cumplimiento de períodos de tiempo en recolección, reporte y diseminación de la información \\
\hline
\end{tabular}

Fuente: $\quad$ Elaboración propia con base en Concha-Eastman y Villaveces (2001).

Con base en esta serie de lineamientos, se aspira no solo a verificar el cumplimiento de los objetivos que inspiran la formulación de un sistema de vigilancia epidemiológica, sino también a garantizar que sus procesos de recolección de datos, de análisis y de reporte sean confiables, buscando mantener la calidad de este.

Finalmente, de los tipos de sistemas de vigilancia epidemiológica que pueden implementarse, destacan los basados en la "revisión de registros institucionales" como aquellos cuya recolección de información se sustenta en el examen periódico de datos provenientes de una o varias instituciones. En razón de tal característica, se subraya la necesidad de identificar adecuadamente las fuentes, así como establecer de manera precisa "la periodicidad, mecanismos de recolección, manejo de la información, evaluación y difusión de los datos [y] variables a seleccionar" (Concha-Eastman y Villaveces, 2001, p.97).

Por otra parte, aquellos sistemas institucionales en los que se recojan los datos partiendo de los casos que las instituciones rutinariamente registran, son calificados como de "recolección pasiva". Respecto a esta variante, se recomienda la confección de formularios de reporte sencillos y fáciles de completar, ello con el propósito de no comprometer su llenado por dificultades de comprensión o falta de interés por parte de quienes los aplican (Concha-Eastman y Villaveces, 2001).

\section{EPIDEMIOLOGÍA SOCIAL: DE LA REALIDAD NUMÉRICA A LA REALIDAD SOCIAL}

Tal y como se indicó en líneas anteriores, en razón de sus antecedentes históricos, la aplicación tradicional o clásica de la epidemiología ha privilegiado los factores numéricos y biológicos en el recuento y análisis de sus temas de estudio, restándole crédito a las fuerzas sociales y a las variables culturales $y$ de comportamiento como parte de su comprensión.

Sin embargo, esta tendencia ha sido discutida por una rama de esta disciplina, definida como epidemiología social, la cual se propone articular una nueva visión de la salud y de la enfermedad en calidad de procesos de producción 
y organización de una sociedad, estrechamente vinculados con conflictos y relaciones de poder (González, 2000). De acuerdo con Krieger (2002), la epidemiología social fue nombrada de esta manera por primera vez en el idioma inglés en 1950, haciendo referencia al estudio de la distribución de la salud, la enfermedad y el bienestar de los grupos a partir de los determinantes sociales.

La epidemiología social se encarga de estudiar cómo las diferentes formas de organización social influyen en el bienestar integral de los individuos, permitiendo con ello, una mayor comprensión del impacto de las desigualdades en el estado de salud de grupos humanos específicos, gracias a la inclusión de la experiencia social de las poblaciones en la etiología tradicional de la salud. De esta manera, se nutre el campo de la epidemiología clásica con perspectivas sociales provenientes de la Sociología, la Economía y la Demografía, con el propósito de comprender y analizar el cómo, dónde y por qué las desigualdades sociales afectan la salud de diversos colectivos humanos (OPS, 2002).

En palabras de González (2000), la epidemiología social busca generar un nuevo tipo de conocimiento que permita "ofrecer alternativas para el cuidado y atención de la salud cuya finalidad no sea de carácter paliativo, sino que modifique las condiciones de fondo y de estructura que están participando en el comportamiento del problema" (p.216); abordaje que contempla inevitablemente el análisis y la deconstrucción de las desigualdades legitimadas culturalmente $y$ que justo subyacen a muchos de los principales problemas de salud pública mundiales, tales como, las múltiples expresiones de violencia contra las mujeres, la población sexualmente diversa, los niños, las niñas, las personas adolescentes, las personas adultas mayores, con discapacidad, migrantes, indígenas, con diferente origen étnico, entre otros.

Así, la epidemiología social aspira a mirar a través de la superficie, más allá de las primeras coordenadas de lectura asentadas en la dimensión biológica o estadística, para apreciar el fondo y proponer vías que transformen ese núcleo tradicional de interpretación de la realidad. De acuerdo con Kaufman y Mezones-Holguín
(2013), se busca trabajar con dos aspectos, la vigilancia y la investigación etiológica; en la primera se "representan los patrones de la enfermedad a lo largo de las dimensiones de la distinción social, tales como las tasas o los riesgos de diferentes desenlaces por categorías de variables como la educación, los ingresos o la etnia" (p. 544). Por su parte, en la investigación etiológica se contrapone el mundo real frente al hipotético, con el propósito de estimar la relación entre el cambio esperado y el resultado, es decir, considerar los efectos sobre variables sociales como programas educativos, ingresos económicos, entre otros.

Para Krieger (2002), algunas de las principales características o principios de la epidemiología social que enriquecen la mera recolección de datos dentro de los sistemas de vigilancia e información, podrían resumirse de la siguiente manera:

$\diamond \quad$ Expresión biológica de la desigualdad social: expone como las personas integran durante la vida sus experiencias de desigualdad, tanto económica como social, y cómo estas producen desigualdades en la salud.

$\diamond \quad$ Teoría ecosocial de la distribución de la enfermedad: integra como las condiciones sociales de las personas y los procesos biológicos producen la salud de la población.

$\diamond \quad$ Derechos humanos y justicia social: reflexiona acerca de cómo el cumplimiento de los derechos humanos promueve la salud de las personas, pero como también la violación de estos puede afectarla, además de que la justicia social permite reconocer a quiénes beneficia $y$ oprime la desigualdad.

$\diamond \quad$ Perspectiva vital: hace referencia a cómo el desarrollo biológico y social de las personas y las condiciones sociales, económicas y culturales reflejan el estado de salud de determinados grupos generacionales.

$\diamond \quad$ Análisis de múltiples niveles: permiten estudiar como las características individuales, contextuales y socioculturales determinan la salud de las personas. 
$\diamond \quad$ Discriminación: los estudios de la epidemiología social que analizan las consecuencias que tiene la discriminación que viven las personas y las poblaciones, buscan reconocer la interseccionalidad en la discriminación que atraviesa a muchos grupos sociales. Para eso reconocen diversos aspectos de la discriminación como el tipo, la forma (legal, ilegal, directa, indirecta), el agente (Estado, instituciones, personas), la manifestación (verbal, física, sexual, patrimonial), la esfera o el espacio en el que se da, el nivel (individual, interpersonal, comunitario, social); así como, la duración en el tiempo y la intensidad a esta exposición. Género y sexo: analiza como los aspectos de la salud están vinculados al género y al sexo, más allá de las expresiones biológicas, pues los roles de género, el sexismo y sus implicaciones desiguales varían dentro de cada sociedad y entre las diferentes sociedades.

$\diamond \quad$ Sexualidades $y$ heterosexismo: la salud de las personas está mediada no solo por las experiencias sexuales, sino también por condiciones sociales como la discriminación, la familia, la economía, etc., a las que se ven expuestas.

$\diamond \quad$ Raza, etnia y racismo: para los estudios de la epidemiología social resulta fundamental considerar las experiencias de racismo vividas por las personas y como esta discriminación racial tiene consecuencias en su salud. Alejándose de los estudios epidemiológicos tradicionales que explicaban las diferencias raciales en la salud de las personas a partir de la genética.

Con la inclusión de estos principios, la epidemiología puede orientarse hacia dimensiones más sociales y culturales, considerando temáticas como la pobreza, la raza, las desigualdades o el género, para ilustrar la diversidad de estrategias metodológicas por medio de las cuales puede buscarse una adecuada elucidación de los datos estadísticos. Dichas dimensiones contribuyen a develar las jerarquías sociales que influyen en el acceso a la salud $y$ pueden originar efectos nocivos en la calidad de vida de las personas $y$ en los procesos psicosociales de grupos humanos específicos (Borrell, 2015).

Otros alcances que se pueden obtener gracias a la utilidad de estos principios en los sistemas de vigilancia epidemiológica son:

$\diamond \quad$ Dar seguimiento a los compromisos adquiridos por el país en materia de prevención, atención y sanción de la violencia.

$\diamond \quad$ Diseñar estrategias y políticas nacionales y locales referidas a la violencia como problema de salud pública.

$\diamond \quad$ Identificar grupos vulnerables o condiciones psicosociales riesgosas frente al fenómeno de la violencia.

$\diamond \quad$ Identificar necesidades en relación con el registro de datos, mantenimiento y sostenibilidad en el tiempo de los sistemas de información.

$\diamond \quad$ Determinar los métodos de registro más efectivos en relación con el tema.

$\diamond \quad$ Justificar la solicitud de cambios legislativos que mejoren la respuesta estatal frente al fenómeno de la violencia.

$\diamond \quad$ Incentivar la cooperación técnica y financiera de agencias internacionales en el abordaje de este problema.

En lo que respecta a la definición de variables a partir de las cuales se registra y analiza la información recolectada, resulta oportuno señalar la importancia de distinguir entre las categorías sexo y género - dadas las confusiones que se presentan en torno a ambos términos-, ello por cuanto la sola mención de la palabra género no conlleva una real comprensión de su acepción, como tampoco la inclusión de una perspectiva de género o de derechos humanos en la formulación del sistema o en su posterior uso. En este sentido, Gómez (2002) aporta la siguiente aclaración:

Género no es sinónimo de sexo. 'Sexo' alude a la diferencia biológica entre el hombre y la mujer, mientras que 'género' se refiere a la construcción social de lo 
"masculino" y lo "femenino" y a la forma como se articulan estas dos construcciones en relaciones de poder. Género tampoco equivale a mujer. El concepto de género no se aplica a la mujer —ni tampoco al hombre-per se, sino a las relaciones de desigualdad entre mujeres $y$ hombres (o entre los ámbitos 'masculinos' $y$ 'femeninos') en torno a la distribución de los recursos, las responsabilidades y el poder (p.455).

Tal clarificación conceptual resulta pertinente, en tanto constituye un aporte que debe ser integrado en todos los sistemas de información y vigilancia, más aún cuando el fin último de estos es dar cuenta de fenómenos que afectan a colectivos sociales - como la violencia- $y$ en consecuencia, deben reconocer el entramado de factores genéricos, generacionales, contextuales y políticos, entre otros, que intervienen en el origen, curso y expresión de estos.

En este sentido, el análisis contextualizado desde el género contribuirá al reconocimiento histórico de las desigualdades existentes entre hombres y mujeres en el acceso $y$ disfrute de la salud. Sin embargo, resulta oportuno ampliar dicha variable de acuerdo con los postulados actuales (Salas y Campos, 2010) que resaltan la importancia de performar las expresiones culturales tradicionales vinculadas con lo femenino y lo masculino; de esta manera se pueden incluir variables en torno a la identidad genérica (cisgénero: la identidad de género coincide con el sexo biológico asignado al nacer; transgénero: la identidad de género no coincide con el sexo biológico asignado al nacer; género no binario: la identidad de género no se define desde las categorías femenino-masculino) y las prácticas sexuales (por ejemplo, la categoría HSH, utilizada para describir a aquellos hombres que tienen sexo con otros hombres, pero que no se autodefinen como homosexuales).

Los sistemas de vigilancia deben ser complementados con otros enfoques que busquen visualizar la diversidad del ser humano, por ejemplo, el enfoque contextual o el enfoque generacional, los cuales enfatizan en otras particularidades como la edad, la etnia o la clase social, para dar cuenta del impacto de la discriminación en la salud de las personas, de acuerdo con distintas condiciones sociales. En concordancia con estos enfoques, otras de las variables socioculturales que pueden incluirse en los sistemas de vigilancia epidemiológicos sobre la violencia, pueden ser la nacionalidad, la orientación sexual y la condición migratoria.

Como puede apreciarse, los aportes de la epidemiología social a los sistemas de información y vigilancia de la salud, enfatizan en la importancia de no solamente presentar indicadores numéricos, sino de entenderles en el marco de un sistema sociocultural específico, planteando líneas de acción derivadas a partir de esta lectura comprensiva. Solamente de esta forma, será posible potenciar la transformación de:

... una distribución desigual de la salud entre el conjunto de la población, tratando de modificar no únicamente la representación estadística del fenómeno, sino las condiciones que hacen que en la problemática sanitaria se expresen las contradicciones $y$ las desigualdades sociales (González, 2000, p. 214).

En este sentido, a pesar de que aún no existen sistemas de información en el país referidos al tema de la violencia que incluyan ampliamente los postulados de la epidemiología social y que sirvan de ejemplo concreto para el análisis de los requerimientos y sugerencias esbozadas en este artículo, se considera que la eventual inclusión de dichos principios fomentaría la promoción de políticas culturales que reconozcan y visualicen la magnitud del problema, así como, la promulgación de políticas públicas que impacten directamente sobre este.

\section{CONCLUSIÓN}

La epidemiología social estudia, desde una perspectiva integral y en un sentido pluralista, la manera en la cual las interacciones sociales afectan la salud de los colectivos humanos. El posicionamiento de esta nueva corriente demanda una mayor correspondencia entre el 
reconocimiento universal que se ha dado a lo social como elemento básico de análisis en el conocimiento de los problemas de salud y las medidas prácticas resolutorias que al respecto se han tomado.

Resulta pertinente destacar que la práctica de la epidemiología social, exige el pasaje de una visión individual a un sentido colectivo de los procesos humanos, cuyos resultados no pueden reducirse a la simple e insuficiente enumeración o clasificación de problemas, sino que debe asumirse un compromiso con el develamiento de las fuerzas económicas, políticas y sociales que determinan el curso de tales problemáticas, es decir, con la visibilización de las desigualdades existentes y el carácter históricamente construido de estas.

En dicho contexto, iniciativas como la inclusión de grupos de edad y la diferenciación por género en las series estadísticas, si bien, son un buen comienzo - en un intento por ampliar los alcances de los sistemas de vigilancia epidemiológica tradicionales-, no aseguran por sí mismas un manejo no reduccionista del dato o de su análisis, en tanto este dependerá de la postura que se asuma en la comprensión de la realidad social y el consiguiente señalamiento de puntos sensibles que requieran intervención.

En síntesis, estas reflexiones permiten visualizar la importancia de que aquellas personas, instancias o comités encargados del diseño, implementación, diseminación y evaluación de los sistemas de información, conozcan a profundidad no solo los requerimientos técnicos que deben ser completados en cada una de las fases de este proceso, sino que además deben contar con información respecto a la trascendencia de entrelazar los productos del sistema con la propuesta teórica y metodológica que propone la epidemiología social. Esta última dirección adquiere especial relevancia en los sistemas vinculados con el fenómeno de la violencia, por cuanto la violencia en sus más diversas manifestaciones no puede ser comprendida en desapego de los sistemas socioculturales $y$ contextos históricos que le validan y propician; de allí la importancia de ir más allá de los meros datos estadísticos.

\section{REFERENCIAS}

Borrell, C. (2015). Epidemiologia social: la persona, la población y los determinantes sociales de la salud. Cuadernos de la Fundación Dr. Antonio Esteve, 32, 33-37.

Concha, A. (2002). Impacto social y económico de la violencia en las Américas. Biomédica, 22(2), 347-361.

Concha-Eastman, A. y Villaveces, A. (2001). Guías para el diseño, implementación y evaluación de sistemas de vigilancia epidemiológica de violencia y lesiones. Washington, DC: Organización Panamericana de la Salud (ops) y Organización Mundial de la Salud (oms).

Drucker, E. (2006). Métodos en Epidemiología Social. (Reseña del libro Methods in Social Epidemiology de Oakes, M. y Kaufman, J. (Eds.), 2006). Medicina Social, 1(3), 108-109.

Gómez, E. (2002). Equidad, género y salud: retos para la acción. Revista Panamericana de Salud Pública, 11(5/6), 454-461.

González, N. (2000). Epidemiología y salud pública frente al proyecto neoliberal en México. Papeles de población, 6(25), 207-225.

Kaufman, J.S. y Mezones-Holguín, E. (2013). Una epidemiología social para América Latina: una necesidad más allá de la Reflexión sobre las inequidades en salud. Revista Peruana de Medicina Experimental y Salud Publica, 30(4), 543-546.

Krieger, N. (2002). Glosario de epidemiología social. Revista Panamericana de Salud Pública, 11(5/6), 480-490.

Larizgoitia, I. (2006). La violencia también es un problema de salud pública. Gac Sanit, 20(1), 63-70.

Lemus, J. (1996). Manual de vigilancia epidemiológica. Washington, DC: Organización Panamericana de la Salud.

Oakes, M. y Kaufman, J. (2006) (Eds.). Methods in social epidemiology. United States of America: John Wiley \& Sons.

Organización Mundial de la Salud (2002). Informe mundial sobre la violencia 
y la salud: resumen. Washington, DC: Organización Panamericana de la Salud.

Organización Panamericana de la Salud. (2001). Guías para el diseño, implementación y evaluación de sistemas de vigilancia epidemiológica de violencia y lesiones. Washington, DC.

Organización Panamericana de la Salud (2002). Introducción a la epidemiología social. Boletín Epidemiológico, 23, (2), 10-13.

Organización Panamericana de la Salud. (2004). La violencia social en Costa Rica. San José, Costa Rica: Ministerio de Salud.
Organización Panamericana de la Salud (2011). Módulos de Principios de Epidemiología para el Control de Enfermedades (segunda edición). Washington DC.

Salas, J. M. y Campos, A. (2010). Explotación sexual comercial: un estudio cualitativo con adolescentes hombres. San José: PANI.

Fecha de ingreso: 15/05/2018 Fecha de aprobación: 28/09/2018 
